\title{
Perception des consommateurs sur la tuberculose humaine et prévalence des agents tuberculeux dans le lait cru de vache au nord de la Côte d'Ivoire
}

\author{
${ }^{*}$ Guillaume B. Gragnon1, Benjamin K. M'Bari², Youssouf B. Kanouté3, Emmanuel Couacy-Hymann1 \\ 1 : National Laboratory for Agricultural Development Support, Korhogo, Côte d'Ivoire \\ 2: Animal Biology, Production and Health Laboratory, Agropastoral Management Institute, Peleforo GON \\ COULIBALY University, Korhogo, Côte d'Ivoire \\ 3 : Freelance Epidemiologist and Public Health Expert, International Consultant/Contractor, Geneva, Switzerland \\ Auteur correspondant : guybiego@gmail.com, mbariben@yahoo.fr
}

Original submitted in on $18^{\text {th }}$ November 2019. Published online at www.m.elewa.org/journals/ on $31^{\text {st }}$ March 2020 https://doi.org/10.35759/JABs.147.1

\section{RÉSUMÉ}

Objectif : Contribuer à l'amélioration de la lutte contre la tuberculose humaine à travers une évaluation des connaissances et des pratiques des consommateurs de lait cru et une évaluation du taux de contamination par Mycobacterium spp du lait de vache mis sur le marché dans la région du Poro.

Méthodologie et résultats : Deux enquêtes ont été réalisées dans le cadre de cette étude. La première qui a porté sur les connaissances, aptitudes et perception a été réalisée à l'aide de questionnaires auprès de 800 consommateurs de lait cru de vaches résidant dans 4 départements de la zone d'étude, à raison de 200 personnes par département. Les participants étaient composés de 540 hommes et 260 femmes avec un âge médian de 30 ans (intervalle : 15 à 70 ans). La seconde enquête a consisté quant à elle, à soumettre au test de Ziehl-Neelsen 304 échantillons de lait cru de vache collectés au pis des mamelles de vaches produisant du lait commercialisé dans les 4 départements d'étude.

Sur des personnes interviewées, seul 4,5\% (36/799) de consommateurs de lait cru de vaches savaient que le lait cru pouvait transmettre la tuberculose et connaissaient les symptômes de cette maladie chez l'homme. Par ailleurs, 36,20\% (289/799) des personnes enquêtées ont déclaré consommer le lait cru quotidiennement et seulement $4,50 \%$ des consommateurs ont indiqué soumettre le lait à un traitement thermique avant sa consommation. Au niveau du lait analysé, environ 22,4\% (68/304) des échantillons testés étaient positifs à Mycobacterium spp.

Conclusion et recommandations : Cette étude a révélé que les connaissances de la population d'étude sur la transmission de la tuberculose via le lait cru sont insuffisantes alors que $22,4 \%$ du lait de vaches locales commercialisé dans la région est contaminé par Mycobacterium spp. Par conséquent, il est recommandé aux autorités sanitaires notamment au programme national de lutte contre la tuberculose de renforcer ses interventions au niveau de l'information et de la sensibilisation des populations locales sur les voies de transmission et les pratiques à risque. Aussi, pour une lutte plus efficace contre cette zoonose, ce programme devra intégrer la dimension santé animale en se basant sur l'approche " one health ».

Mots-Clés : Tuberculose, lait de vache, consommateur, nord Côte d'Ivoire, Mycobacterium spp 


\section{ABSTRACT}

Objective : Contribute to reducing the impact of Mycobacterium bovis infections on humans and animals health and well-being in the Poro region.

Methodology and results : Surveys were carried out using questionnaires administered to 800 consumers of raw cow milk in northern Côte d'Ivoire. These consumers consisted of 540 men and 260 women with median age of 30 years (from 15 to 70). Additionally, three hundred four (304) cow's milk samples were tested by Ziehl Neelsen staining method. About $36.20 \%$ of the participants consumed milk daily, whilst only $4.50 \%$ of them knew the most common symptoms of human tuberculosis. Few respondents knew that tuberculosis contaminated raw milk could transmit tuberculosis to humans. Of 304 milke samples tested 22,4\% were positive for Mycobacterium spp.

Conclusion: The study found major lack of awareness on the transmission of tuberculosis from cattle to humans through the consumption of contaminated cow milk. The authors recommend the national tuberculosis control program authorities in Côte d'Ivoire to focus more on public education and awareness campaigns in northern Côte d'Ivoire.

Keywords: Tuberculosis, cow's milk, consumer, northern Côte d'Ivoire, Mycobacterium bovis

\section{INTRODUCTION}

La tuberculose humaine est une maladie contagieuse transmise par des souches de bactéries du complexe Mycobacterium tuberculosis ou bacilles de Koch. Cependant, les travaux de Müller et al. (2013) ont rapporté la part importante de la tuberculose zoonotique qui a été longtemps sous-estimée, dans la tuberculose humaine. Cette tuberculose zoonotique est une forme de tuberculose transmissible à l'homme, due à Mycobacterium bovis, agent causal de la tuberculose bovine, et appartenant au complexe M. tuberculosis (OMS/FAO/OIE, 2017). Elle affecte très souvent d'autres organes que les poumons lors de la tuberculose extra-pulmonaire et dans de nombreux cas ne peut être différenciée cliniquement de la tuberculose à $M$. tuberculosis. L'homme est contaminé par la tuberculose zoonotique essentiellement lors de la consommation de viandes, du lait ou des produits laitiers contaminés. La tuberculose zoonotique est aussi une maladie professionnelle car la transmission de $M$. bovis est possible par voie aérienne aux personnes qui ont un contact régulier et étroit avec des animaux infectés ou leurs produits (Cosivi et al., 1998 ; Dankner et Davis, 2000 ; Mfinanga et al., 2003 ; Jenkins et al, 2011; Michel et al., 2015). Cependant, les sujets les plus sensibles sont les individus VIH positifs. Ces sujets excrètent des quantités relativement élevées de micro-organismes, favorisant ainsi la transmission interhumaine ou homme-animal (Regassa et al., 2009). Aussi, les cultures, les coutumes et la méconnaissance de la maladie jouent un rôle important dans la persistance et la propagation de la tuberculose à $M$. bovis, constituant souvent des obstacles majeurs à l'application des programmes de contrôle de la maladie (Chillio et al., 2002 ; Mfinanga et al., 2003 ; Ayele et al., 2004 ; Imorou et al., 2004). Dans les pays où la maladie est sous contrôle, les vaches continuent d'éliminer les bactéries du complexe tuberculosis dans le lait. Au Royaume-Uni, Grant et al.(2002) ont détecté des bactéries du complexe tuberculosis dans 7,8\% de lait cru de vache et $11,8 \%$ de lait pasteurisé. Au Bresil, Pardo et al. (2001) ont rapporté la présence de Mycobacterium spp dans 10\% d'échantillons de lait collecté dans les élevages. Et, la spécification des membres de ce complexe par la chromatographie sur couche mince a revelé la présence de Mycobacterium bovis dans 5,26\% des échantillons de lait. Par contre dans la majorité des pays en voie de développement, la maladie est endémique. En Inde et au Népal notamment, Jha et al. (2007) ont rapporté que respectivement 12 et $31 \%$ des vaches produisaient du lait contaminé par Mycobacterium bovis. Au Bénin, Farougou et al. (2011) et Vikou et al. (2018) ont rapporté respectivement des prévalences de $16 \%$ et $36,81 \%$ de Mycobacterium bovis dans le lait de vache. Par ailleurs, Mycobacterium bovis a été 
isolé chez des patients souffrant de la tuberculose avec des prévalences variables dans de nombreux pays. C'est le cas au Mexique où Portillo-Gomez et al. (2011) ont obtenu une prévalence de $28 \%$ contre $10,3 \%$ et $5,3 \%$ respectivement en Inde et en Turquie (Prasad et al., 2005 ; Bayraktar et al., 2011). Au Nigeria, Cadmus (2007) a rapporté des cas de Mycobacterium bovis dans la tuberculose humaine. Selon cet auteur, les prévalences étaient de 3,1\% pour la tuberculose pulmonaire et de $11,1 \%$ pour la tuberculose extra-pulmonaire. Par contre au Ghana, Addo et al. (2007) ont rapporté une prévalence de $3 \%$ de tuberculose pulmonaire humaine causée par Mycobacterium. bovis. D'autres auteurs ont rapporté des prévalences respectives de la tuberculose à Mycobacterium bovis de 15\% au Nigeria (Mawak et al., 2006), de 16\% en Tanzania (Kazwala et al., 2001 ) et $17 \%$ en Ethiopie (Kidane et al., 2002). D'après les estimations de I'OMS, 147000 nouveaux cas de tuberculose zoonotique ont été recensés chez les humains avec 12500 décès dans le monde en 2016 (OMS, 2017). Par contre, en Côte d'Ivoire 21307 cas de tuberculose humaine dont 20939 nouveaux cas et rechutes ont été enregistrés à l'échelle nationale en 2017. Parmi ces cas, $80 \%$ des malades étaient affectés par la forme pulmonaire de la maladie contre $20 \%$ de malades souffrant des formes extra-pulmonaires. Aussi, compte tenu de l'importance de cette maladie et de son évolution, les espèces du genre Mycobacterium ont été classées à la première place sur la liste de priorisation des maladies zoonotiques les plus préoccupantes pour la Côte d'Ivoire (CDC-USAID, 2017). Cependant, malgré l'importance médicale

\section{MATERIEL ET METHODES}

Zone d'étude: Situé entre $8^{\circ} 26$ et $10^{\circ} 18$ degré de latitude Nord et $5^{\circ} 17$ et $6^{\circ} 19$ de longitude Ouest, la région du Poro se trouve dans le nord de la Côte d'Ivoire (Figure 1). Les limites géographiques de la zone d'étude le Mali au nord se trouve, les régions du Tchologo et du Hambol à l'Est, la région du Béré au Sud et la région de la Bagoué à l'Ouest. Le climat est de type soudano-guinéen et comporte deux saisons. La saison sèche qui s'étend de Novembre à Avril est très de la tuberculose dans le pays, peu de données sont disponibles à ce jour sur la tuberculose zoonotique en Côte d'Ivoire. Hormis les rapports annuels de la Direction des Services Vétérinaires (DSV) sur les cas de tuberculose à partir des saisies aux abattoirs, Cissé et al. (2008) ont rapporté une prévalence de $4 \%$ de tuberculose sur les bovins traités à l'abattoir d'Abidjan. Cependant les travaux de ces auteurs ont porté sur des bovins provenant essentiellement des pays sahéliens tels que le Mali, le Burkina Faso voire le Niger. Au niveau du district sanitaire des savanes 591 cas, toutes formes confondue de tuberculose humaine ont été rapportés en 2013 par les services étatiques. Sur cet ensemble, 421 (71\%) cas étaient de formes contagieuses (tuberculose pulmonaire) et $170(29 \%)$ cas de formes extrapulmonaires (Anonyme, 2013). Dans ces circonstances, sachant que la protection de la population humaine doit être envisagée en diminuant le risque de contamination à partir du cheptel infecté, il convient d'une part, d'avoir une meilleure connaissance des pratiques à risque pour l'extension de la maladie; et d'autre part, d'identifier les facteurs favorisant la persistance de la tuberculose zoonotique au sein de la population. C'est dans ce cadre que cette étude exploratoire sur la tuberculose a été initiée. Cette étude vise à contribuer à l'amélioration de la lutte contre la tuberculose humaine à travers une évaluation des connaissances et des pratiques des consommateurs de lait de vaches locales et une évaluation du taux de contamination du lait de vache mis sur le marché dans la région du Poro par Mycobacterium spp.

marquée par l'harmattan entre Décembre et Janvier et une période de fortes chaleurs entre Mars et Avril. L'harmattan souffle du Nord à l'Est pendant plus de trois (3) mois. La saison des pluies s'étend, quant à elle, de Mai à Octobre avec une pluviométrie maximale en Juillet et Août. La moyenne pluviométrique annuelle se situe entre $1200 \mathrm{~mm}$ et $1400 \mathrm{~mm}$. La température moyenne est de $26,5^{\circ} \mathrm{C}$ pour une température souvent supérieure à $40^{\circ} \mathrm{C}$, avec des températures basses 
atteignant $21^{\circ} \mathrm{C}$ à $14^{\circ} \mathrm{C}$ de Décembre à Février. L'humidité maximale est de 83,1\% (Sib, 2013).

Collecte des données: La collecte des données a consisté en une enquête sur les connaissances, les attitudes et les pratiques (CAP) réalisée auprès des consommateurs de lait cru de vache et à la recherche des bacilles alcoolo-acido-résistants (BAAR) dans des échantillons de lait cru de vaches destiné à être commercialisé dans la zone d'étude. L'enquête CAP a été réalisée à l'aide d'un questionnaire standardisé administré par passage unique auprès de 799 consommateurs de lait de vaches locales dont 200 provenaient des départements de Korhogo, de Sinématiali, de Dikodougou et 199 de M'Bengué. Dans chaque département, les consommateurs résidant dans les chefs-lieu de département ont été choisis de façon aléatoire, sans distinction d'âge, de sexe, de religion, de classe sociale et d'ethnie. Le questionnaire utilisé comportait des informations sur le profil sociodémographique des consommateurs interviewés, leurs habitudes de consommation du lait de vache locales et leurs connaissances sur la tuberculose (voies de transmission et symptômes) et les pratiques de consommation de lait de vaches locales. Pour l'analyse du lait, 304 échantillons de lait cru ont été prélevés sur 304 vaches locales dont la production laitière est commercialisée dans la zone d'étude. Pour ce faire, 76 vaches locales issues de 5 fermes péri-urbaines choisies de façon aléatoire dans chacun des 4 chefs- lieux de départements. Dans ces élevages, des vaches également allaitantes ont été choisies de façon aléatoire sans distinction d'âge et de stade de lactation. Le seul critère d'inclusion des vaches était l'exploitation commerciale de leur lait. Pour chaque vache retenue pour cette étude, un échantillon de lait a été collecté dans un tube stérile au moment de la traite matinale, codifié. L'ensemble des échantillons regroupés après chaque collecte ont ensuite été transporté sous glace au Laboratoire National d'Appui au Développement Agricole (LANADA) de Korhogo pour des analyses. Au laboratoire, chaque échantillon de lait a été centrifugé à 3000 tours par minute pendant 10 minutes. A partir de la crème obtenue, un frottis a été réalisé, séché et fixé à l'éthanol absolu à $90^{\circ}$. La coloration de Ziehl a été réalisée conformément à la technique décrite par Macander-Golcz (1963).

Analyse statistique: après le dépouillement des questionnaires, une analyse descriptive des différentes variables en lien avec les connaissances et pratiques de la population cible sur la tuberculose a été réalisée. Au niveau des échantillons de lait, les résultats d'analyses de laboratoire ont permis de déterminer la prévalence de Mycobacterium spp dans la zone d'étude. Les prévalences de l'infection dans les différents départements ont été comparées à l'aide du test de $\mathrm{Khi}^{2}$. L'ensemble des analyses statistiques a été réalisé à l'aide du logiciel XLSTAT (version 2019. 3.2.61545) au seuil de significativité de $5 \%$. 


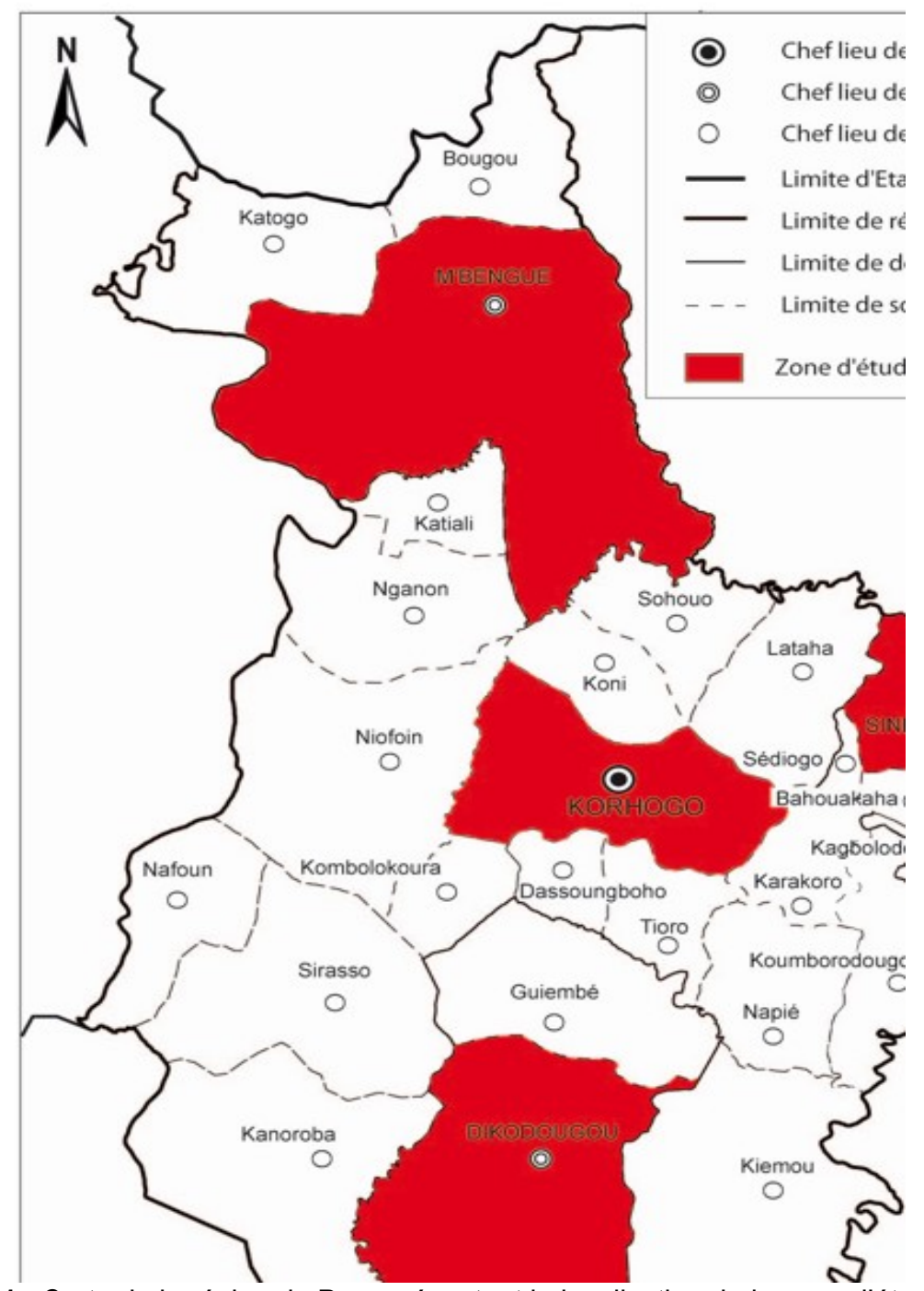

Figure 1 : Carte de la région du Poro présentant la localisation de la zone d'étude

\section{RESULTATS}

Profil sociodémographique des consommateurs: Les résultats de l'enquête ont montré que sur les 799 consommateurs de lait de vaches locales enquêtés, $67,7 \%$ étaient des hommes et $32,3 \%$ des femmes. La différence statistique entre ces 2 sous-populations était significative au seuil $(p<0,0001)$. Les consommateurs de lait de vaches locales interviewés au cours de cette étude avaient un âge compris entre 15 et 70 ans avec un âge médian de 30 ans. Cependant, il a été noté que près des $2 / 3$ de ces consommateurs $(61,6 \%)$ avaient un âge compris entre 19 et35 ans. La variation de leur effectif selon les tranches d'âge était statistiquement significative avec $p<0,0001$ (Tableau 1). Selon les résultats de l'enquête environ $56,7 \%$ des consommateurs interviewés étaient analphabètes avec une majorité $(65 \%)$ résident en zone urbaine (tableau $1)$. 
Tableau 1 : Profil sociodémographique des consommateurs de lait de vaches locales de la région du Poro

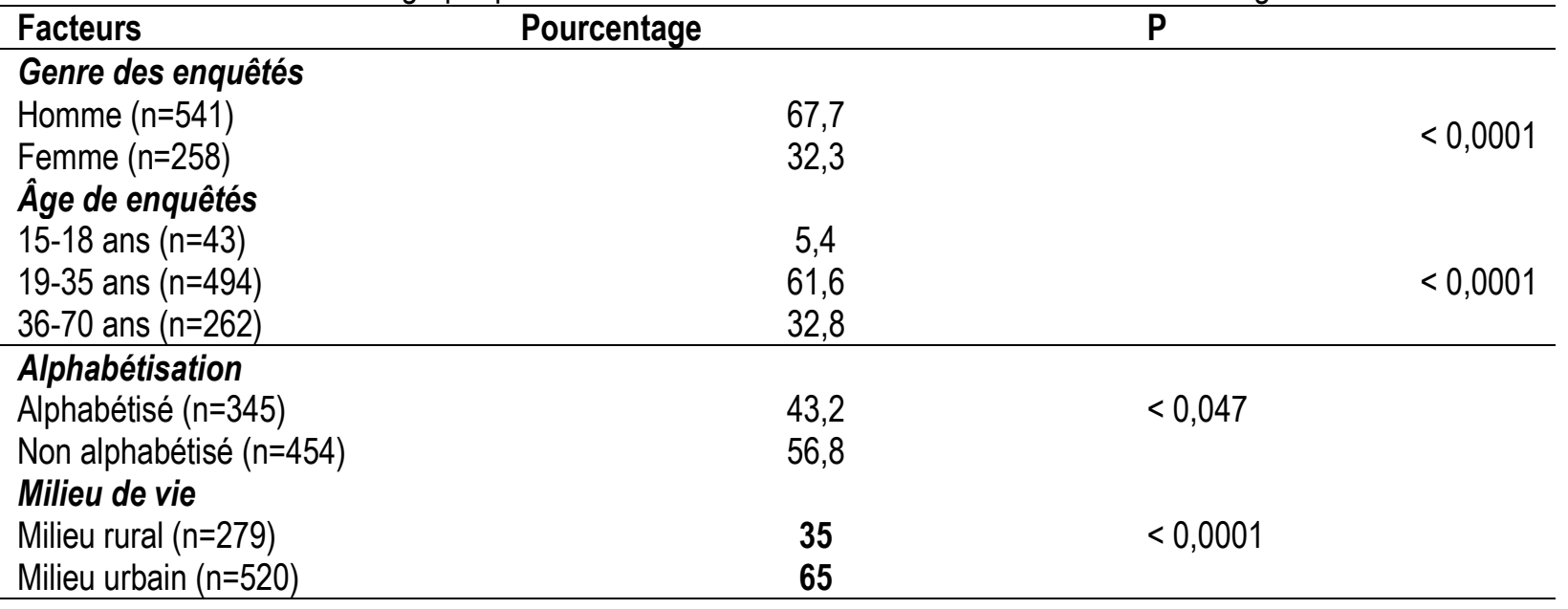

Habitudes de consommation de lait de vaches locales et Niveaux de connaissances sur la tuberculose: Concernant les habitudes de consommation $36,2 \%$ des personnes interviewées consommaient le lait cru de vache quotidiennement tandis que environ $63,8 \%$ le consommaient de façon irrégulière c'est-à-dire 1 à 6 jours par semaine (tableau
2). La différence observée au niveau de la fréquence de consommation est statistiquement significative $(p<$ 0,0001 ). Au niveau du mode de consommation du lait, seuls $4,5 \%$ des consommateurs ont déclaré chauffer le lait avant consommation. Cependant la température et la durée de chauffage du lait n'étaient pas toujours précisées.

Tableau 2: Habitudes de consommation de lait de vaches locales et Niveaux de connaissances sur la tuberculose

\begin{tabular}{lccc}
\hline \multicolumn{1}{c}{ Facteurs } & & Pourcentage & $\mathbf{p}$ \\
\hline Fréquence de consommation du lait de vaches locales & & & \\
Journalière & 289 & 36.2 & $<0,0001$ \\
Irrégulière & 510 & 63.8 & \\
Mode de consommation du lait de vaches locales & & & \\
$\quad$ Lait consommé après chauffage & 36 & 4,5 & $<0,0001$ \\
$\quad$ Lait consommé à l'état cru (sans traitement) & 763 & 95,5 & \\
\hline Connaissance des symptômes majeurs de la tuberculose & 36 & 4,5 & $<\mathbf{0 , 0 0 0 1}$ \\
Connait & 763 & 95,5 & \\
Ne connait pas & & & \\
Connaissance du mode de transmission de la tuberculose par le lait & 36 & 4,5 & $<0,0001$ \\
Connait & 763 & 95,5 & \\
Ne connait pas & & & \\
\hline
\end{tabular}

Cette enquête a révélé que la plupart des consommateurs de lait de vaches locales $(95,5 \%)$ ignoraient que le lait pouvait transmettre la tuberculose à l'homme. Sur le plan clinique, seulement $4,5 \%$ des participants savaient énumérer des symptômes majeurs de la tuberculose. D'une façon générale, la tuberculose est très peu connue des consommateurs de lait de vaches locales de la zone d'étude. En effet, la proportion des consommateurs n'ayant pas de connaissances sur la tuberculose humaine était significativement plus grande que celle des consommateurs connaissant les symptômes de cette maladie et son mode de contamination par le lait $(p<0,0001)$.

Prévalence globale de Mycobacterium spp dans le lait de vaches issu d'élevage de la région du Poro: Sur 304 prélèvements de lait de vaches collectés dans les quatre départements de la région du Poro, 22,4\% (68/304) se sont révélés positifs à Mycobacterium spp (Tableau 4). La prévalence de la tuberculose a semblée plus élevée dans le lait issu des départements de Sinématiali $(29 \%)$ et de Korhogo $(23,7 \%)$ que dans les 
autres départements. Aussi, toutes les localités investiguées ont possédé des laits positifs au test de Ziehl-Neelsen révélant ainsi que les Bacilles alcooloacido résistants circulent dans les départements de la région du Poro. Cependant, il est à noter que les prévalences déterminées dans les départements retenus pour cette étude ne diffèrent pas significativement $(p>0,05)$.

Tableau 4 : Fréquence de Mycobacterium sp dans le lait cru de vache par localité dans la région du Poro

\begin{tabular}{lccc}
\hline Localités & Positifs & Pourcentage & p-value \\
\hline Korhogo $(n=76)$ & 18 & & \\
Dikodougou $(n=76)$ & 12 & 23,7 & 0,574 \\
Napié $(n=76)$ & 16 & 15,8 & \\
Sinématiali $(n=76)$ & 22 & 21 & \\
Total $(304)$ & 68 & 29 & \\
\hline
\end{tabular}

\section{DISCUSSION}

Face aux évidences sur l'évolution de nouveaux cas de tuberculose en Côte d'Ivoire en général, cette étude a été conduite pour évaluer les connaissances sur la tuberculose et les habitudes de consommation du lait de vaches locales d'une part; et d'autre part pour estimer la prévalence de la tuberculose bovine à travers une analyse du lait de vaches locales. Elle a été conduite dans le nord du pays où se trouvent environ $60 \%$ des élevages bovins. II ressort de cette enquête que les consommateurs de lait de vaches locales méconnaissent la tuberculose humaine et ignorent que cette maladie est transmissible par la consommation de lait cru de vache contaminée. Dans la zone d'étude, la consommation du lait de vache locale se fait préférentiellement sans traitement thermique (à l'état $\mathrm{cru}$ ) alors que toutes les localités enquêtées possèdent des vaches dont le lait contient des bacilles de la tuberculose. Sur les 799 consommateurs enquêtés, $67,7 \%$ étaient des hommes et $32,3 \%$ des femmes. La proportion d'homme consommateurs de lait était statistiquement plus élevée que celle des femmes. L'âge médian était de 30 ans et les extrêmes de 15 et 70 ans. Ces résultats sont très proches de ceux de l'enquête réalisée en 2016 sur la population de consommateurs de lait de la zone de Niamey au Niger (Anonyme, 2016). Selon cette étude l'âge moyen des consommateurs était de 33 ans avec un minimum de 14 et un maximum de 65 ans. Par ailleurs, dans cette étude, toutes les personnes enquêtées étaient issues $\mathrm{du}$ milieu urbain. Cependant, contrairement à notre étude, $90 \%$ de personnes ciblées dans cette étude étaient instruites. En Algérie, Mamine et al. (2016) ont également rapporté dans leur étude sur la qualité de lait que les consommateurs de lait de vaches locales étaient composés de $53,8 \%$ d'hommes et $46 \%$ de femmes pour un âge variant entre 18 et 75 ans. Les travaux de ces auteurs ont révélés que $56,8 \%$ des consommateurs étaient analphabètes et la plupart $(65 \%)$ étaient issus de zones urbaines. Selon le rythme de consommation du lait de vaches locales, $36,2 \%$ des personnes interviewées au cours de notre étude ont indiqué consommer le lait de vaches quotidiennement. Dans 95,5\% des cas, les consommateurs ne pasteurisaient pas le lait avant consommation et ne connaissaient pas la tuberculose. Selon FAO and ECOWAS (2016), cette attitude s'observe généralement en milieu rural où le niveau de transformation du lait est très faible si bien qu'il est consommé presqu'en l'état naturel. Au Niger, l'étude de Boukary et al. (2012) a montré que $85 \%$ à $94 \%$ des enquêtés consommaient du lait cru et des produits dérivés de lait cru non pasteurisés. Les résultats de nos travaux montrent que les pratiques alimentaires en lien avec la consommation de lait de vaches locales sont à risque. Ces risques sont d'autant plus importants que la prévalence de Mycobacterium spp. dans le lait des vaches locales est élevée (22,4\%). Cette analyse est confortée par le fait que la prévalence de la tuberculose est généralement très élevée en Afrique subSaharienne. Cette observation a été également confirmée par Boukary et al. (2012). En effet ces auteurs qui ont mené une étude sur la tuberculose au Niger ont rapporté que $22,8 \% ; 30,5 \%$ et $43,1 \%$ des consommateurs de leurs trois sites d'étude ont déclaré connaitre des personnes de leur voisinage qui présentaient des signes similaires à ceux de la tuberculose. La prévalence de Mycobacterium spp. dans le lait des vaches élevées dans la région des savanes est supérieure à celles rapportées par Farougou et al. (2011) au Bénin (16\%), Jha et al. (2007) en Inde (12\%) et anonyme (2006) en France $(7 \%)$. Cependant, la prévalence de la tuberculose 
bovine dans notre zone d'étude est bien inférieure à celles de $36,81 \%$ relevée au Bénin par Vikou et al.

\section{CONCLUSION}

Selon cette étude le niveau de connaissance sur la tuberculose est insuffisant. Ce qui pourrait expliquer le fait que les consommateurs ont des habitudes alimentaires qui les exposeraient à cette maladie à travers la consommation de lait cru. Le risque de contamination par le lait est si élevé que $22,4 \%$ de lait de vache testés dans la zone d'étude se sont révélés positifs à Mycobacterium spp. C'est le lieu d'interpeller les autorités en charge du programme national de lutte

\section{RÉFÉRENCES BIBLIOGRAPHIQUES}

Addo KK, Owusu-Darko K, Yeboah-Manu D, Caulley P, Minamika M, Bonsu F, Leinhardt C, Akpedonu P, Ofori-Adjei D. 2007. Mycobacterial species causing pulmonary tuberculosis at Korle $\mathrm{Bu}$ teaching hospital, Accra, Ghana. Ghana Medical Journal, 41, 52-57.

Anonyme. 2013. Rapport d'activités annuelles du Centre Anti Tuberculeux (CAT) de Korhogo. http://news.abidjan.net/h/495344.html.

(Consulté le 27 juillet 2019).

Anonyme. 2016. Etude du marché des produits laitiers $100 \%$ nigériens à base du lait local. Rapport d'enquête. P39.

Ayele W Y, Neill S, D, Pavlik I, Zinsstag J. et Weiss M. G. 2004. Bovine tuberculosis : an old disease but a new threat to Africa International journal of tuberculosis and lung disease 8 (8): 924937p.

Bayraktar B, Bulut E, Barış A B, Toksoy B, Dalgıc N, Celikkan C, \& Sevgi D. 2011. Species Distribution of the Mycobacterium tuberculosis Complex in Clinical Isolates from 2007 to 2010 in Turkey: a Prospective Study. Journal of Clinical Microbiology, 49(11), 3837-3841. doi:10.1128/jcm.01172-11.

Boukary A R, Thys E, Rigouts L, Matthys F, Berkvens D, Mahamadou I, Yenikoye A, Saegerman C. 2012. Risk Factors Associated with Bovine Tuberculosis and Molecular Characterization of Mycobacterium bovis Strains in Urban Settings in Niger. Transboundary and Emerging Diseases. pp102-114.

Cadmus S. 2007. Bovine tuberculosis in Nigeria. In: Proceedings of the 1st meeting «African Bovine TB Network : Effective management of bovine tuberculosis in Africa : Towards
(2018) et de 31\% au Népal par de Sinha (1994).

contre la tuberculose sur d'une part, la nécessité de renforcer les actions de sensibilisation et d'information sur la tuberculose; et d'autre part, d'intégrer dans ce programme des actions en direction de la lutte contre la tuberculose animale. Pour ce faire, la mise en œuvre de l'approche " One health » parait indispensable pour renforcer durablement la lutte contre la tuberculose en Côte d'Ivoire.

adapted control policy ». Bamako, Mali, 26-29 juin 2007, 5-6.

CDC-USAID. 2017. Priorisation one health des maladies zoonotiques pour un engagement multisectoriel. Côte d'Ivoire. Rapport. 20 P.

Chillio A, Elhadji D, Moumouni A, Souley A. 2002. Autour de la contagion de la transmission, et de la prévention : notions populaires hausa et songhay-zarmamai. Etudes et Travaux $n^{\circ} 7$. Laboratoire d'études et recherches sur les dynamiques sociales et le développement local (LASDEL). Niamey, Niger, 46p.

Cissé B, N'guessan K, Ekaza E, Soro, Aka, Dosso M. 2008. Isolement de Mycobacterium bovis des lésions tuberculeuses chez les bovins à l'abattoir d'Abidjan Port-Bouët (Côte d'Ivoire). 199-204p.

Cosivi O, Grange JM, Daborn CJ, 1998. Zoonotic tuberculosis due to Mycobacterium bovis in developing countries. Emerg Infect Dis ; 4: 5970.

Dankner W M et Danvis C E. 2000. Mycobacterium bovis as a significant cause of tuberculosis in children residing along the United States Mexico border in the Baja California region. Pediatrics, 105-79p.

ENV. 2006. Ecoles Nationales Vétérinaires Françaises, Maladies Contagieuses. La tuberculose animale, document polycopié à l'usage des étudiants vétérinaires. $13 \mathrm{p}$.

FAO and ECOWAS. 2016. Revue des filières bétail/viande \& lait et des politiques qui les influencent au Burkina Faso. I5261F/1/04.16. 73 p. file:///C:/Users/DRGRAG 1/AppData/ Local/Temp/9789242513042-fre.pdf (consulté le 02 juin 2019). 
Farougou S, Legba Gbenou A M, Aplogan L G. 2011. Fréquence de la tuberculose bovine dans le lait et la viande dans le département du borgou au bénin. In Acte du 3 e Colloque des sciences, cultures et technologies de I'UACBénin (309-322)

Imorou A., Olivier JP., De S. 2004. La tuberculose à gaya : approche socioanthropologique. Etudes et Travaux $n^{\circ}$ 25. LASDEL, Niamey, Niger, 20p.

Grant I. R., Ball H. J., Rowe M. T. 2002. Incidence of Mycobacterium paratuberculosis in Bulk Raw and Commercially Pasteurized Cows' Milk from Approved Dairy Processing Establishments in the United Kingdom. Applied And Environmental Microbiology, May 2002, p. 2428-2435 Vol. 68, No. 5 00992240/02/\$04.00_0 DOI: 10.1128/AEM. 68.5.2428-2435.2002. American Society for Microbiology.

Jenkins A O, Cadmus S I B, Venter E H, Pourcel C, Hauk Y, Vergnaud G, Godfroid J. 2011. Molecular epidemiology of human and animal tuberculosis in Ibadan, Southwestern Nigeria. Veterinary Microbiology, 151, 139-147p.

Jha VC, Morita Y, Dhakal M, Besnet B, Sato T, Nagal A, Kato M, Kosawa K, Yamamoto S, Kimura H. 2007. Isolation of Mycobacterium spp. from milking buffaloes and cattle in Nepal. Journal of Veterinary Medical Science. 69:819-825.

Kazwala RR, Daborn CJ, Sharp JM, Kambarage DM, Jiwa SF, Mbembati NA. 2001. Isolation of Mycobacterium bovis from human cases of cervical adenitis in Tanzania: a cause for concern. Tuber Lung Dis ; 5: 87-91.

Kidane D, Olobo J O, Habte A, Negesse Y, Aseffa A, Abate G, Harboe M. 2002. Identification of the Causative Organism of Tuberculous Lymphadenitis in Ethiopia by PCR. Journal of Clinical Microbiology, 40(11), 4230-4234. doi:10.1128/jcm.40.11.4230-4234.2002

Macander-Golcz W. 1963. Recherches sur la présence de " mycobactéries", types hominis et bovis, dans le lait de marché à Varsovie. Le Lait, INRA Editions, 43 (421_422), pp.30-35.

Mamine F, Montaigne E, Boutonnet J P. 2016. Perception de la qualité des produits laitiers et comportement du consommateur algérien. Économie Rurale 35. (49-65).

Mawak J, Gomwalk N, Bello C, Kandakai-Olukemi Y. 2006. Human pulmonary infections with bovine and environment (atypical) mycobacteria in Jos, Nigeria. Ghana Med J ; 40: 132-36).

Mfinanga S G, Morkve O, Kazwala R, Cleavland S, Kunda J, Sharp M J. 2004. Mycobacterial adenitis: role of Mycobacterium bovis, nontuberculosis Mycobacteria, HIV infection and risk factors in Arusha, Tanzania. E Afr Med J., 171-178p.

Michel AL, Geoghegan C, Hlokwe T, Raseleka K, Getz WM, Marcotty T. 2015. Longevity of Mycobacterium bovis in raw and traditional souring milk as a function of storage temperature and dose. PLoS One; 10: e0129926).

Müller B, Dürr S, Alonso S, et al. 2013. Zoonotic Mycobacterium bovis-induced tuberculosis in humans. Emerg Infect Dis ; 19: 899-908

OMS, FAO et OIE. 2017. Feuille de route pour la tuberculose zoonotique. Rapport. $24 \mathrm{p}$.

Pale E. 2006. Analyse de la consommation du lait et des produits laitiers : Cas de la ville de BoboDioulasso. Mémoire diplôme d'ingénieur du développement rural option sociologie et économie rurales. $45 \mathrm{P}$.

Pardo, R. B. ; Langoni, H.; Mendonça, L. J. P.; Chi, K. D. Isolation of Mycobacterium spp. in milk from cows suspected or positive to tuberculosis. Braz. J. vet. Res. anim. Sci. São Paulo, v. 38, n. 6, p. $284-287$

Portillo-Gomez L, Sosa-Iglesias EG. 2011. Molecular identification of Mycobacterium bovis and the importance of zoonotic tuberculosis in Mexican patients. Int J Tuberc Lung Dis. 15: 1409-14).

Prasad H K, Singhal A, Mishra A, Shah N P, Katoch V M, Thakral S, Acharya S K. 2005. Bovine tuberculosis in India: Potential basis for zoonosis. Tuberculosis, 85(5-6), 421-428. doi:10.1016/j.tube.2005.08.005

Regassa A, Medhin G, Ameni G. 2009. Bovine tuberculosis is more prevalent in cattle owned by farmers with active tuberculosis in central Ethiopia. The Veterinary Journal, 178, 119$125 p$.

SIB 0. 2013. Analyse de la diversité et de la dynamique des systèmes agraires : cas des exploitations agropastorales de la région de Korhogo (Côte d'Ivoire). Mémoire de Master Biologie et Productions Animales. UNA, Unité de Formation et de Recherche en Sciences de la Nature. P 96 
Sinha RN. 1994. Mycobacterium tuberculosis. In : The significance of pathogenic microorganisms in raw milk, éds. Hahn, G. \& Heeschen, W. pp.113-140. Brussels (Belgium) : International Dairy Federation.

Vikou R, Aplogan LG, Ahanhanzo C, Baba-Moussa L, Gbangboche AB. 2018. Prévalence de la brucellose et de la tuberculose chez les bovins au Bénin. Int. J. Biol. Chem. Sci. 12(1) : 120128 